complaints and address the mood issues to reduce burden of memory dysfunction in patients with epilepsy.

\section{A0026: Children (12-18 Years Age) of Women with Epilepsy Have Lower Intelligence, Attention, and Memory: Observations from the Kerala Registry of Epilepsy and Pregnancy \\ Manna Jose, ${ }^{1}$ Veena P., ${ }^{1}$ Nikita Susan Jacob, ${ }^{1}$ Sanjeev V. Thomas $^{1}$ \\ ${ }^{1}$ Department of Neurology, Sree Chitra Tirunal Institute for Medical Sciences and Technology, Trivandrum, Kerala, India}

Objective: We aimed to study the cognitive outcome of 12- to 18-year old children of women with epilepsy (WWE).

Methods: Children of WWE (12-18 years) under follow-up in Kerala Registry of Epilepsy and pregnancy $(n=86)$ were evaluated with Wisconsin Card Sorting Test (WCST), trail making test (TMT), Rey auditory verbal learning test (RAVLT), and Rey-Osterrieth complex figure test (ROCFT).

Results: There were 41 women with generalized epilepsy (47.7\%) and 45 with localization-related epilepsy (52.3\%). Antiepileptic drugs (AEDs) exposure was as follows: carbamazepine (26), valproate (21), phenytoin (five), phenobarbital (three), polytherapy (25), and six were unexposed. The full-scale IQ (FSIQ) and higher order executive functioning of children of these 86 WWE showed significant difference with AED exposure. The FSIQ mean \pm SD for different AEDs were phenobarbital: (73.6 \pm 14$)$, phenytoin: $(87.7 \pm$ 22.1), carbamazepine: (96.4 \pm 8.2 ), valproate: (93.6 \pm 13.6 ). The FSIQ for those exposed to phenobarbital was significantly ( $p=0.045$ ) lower than others, whereas those children unexposed to AEDs had high FSIQ scores (mean $=97.92, \mathrm{SD}=$ $10.72 ; p=0.028$ ). Higher order executive functioning, specifically the ability to maintain and shift set was found to be low in children exposed to phenobarbital and valproate monotherapy compared with no AED exposure $(p=0.049)$.

Conclusion: IQ and higher order executive functioning were significantly lower for 12- to 18 - year-old children of WWE exposed to different AEDs when compared with nonexposed children. Thus, antenatal AED exposure is an important predictor of low FSIQ and higher order executive functioning.

A0027: Effect of Antidepressants (Sertraline, Escitalopram) in Combination with Antiepileptic Drugs (Sodium Valproate, Levetiracetam) on Seizures, Cognitive Impairment, and Oxidative Stress in Rats

Md. Hussain, ${ }^{1}$ Jatinder Katyal, ${ }^{1 *}$ Haroon Rashid, ${ }^{1}$ Yogendra K. Gupta'

${ }^{1}$ Neuropharmacology Laboratory, Department of Pharmacology, All India Institute of Medical Sciences, New Delhi, India

Objectives: Depression is a major comorbidity in persons with epilepsy ( $p \mathrm{WE}$ ). The effect of antidepressants sertraline (SRT) and escitalopram (ESC) in combination with sodium valproate (SV) and levetiracetam (LEV) on seizure and cognitive impairment in rats was studied.
Methods: The male Wistar rats (200-250 g) were trained for Morris water maze (MWM), passive avoidance $(p \mathrm{~A})$ and elevated plus maze (EPM) test and baseline trials conducted before subjecting to pentylenetetrazole ( $p \mathrm{TZ}$ )induced kindling. SV and LEV were used in combination with SRT and ESC to evaluate effect on seizure development, cognition, and biochemical parameters. SRT (25 mg/kg), ESC (15 mg/kg), SV (150 mg/kg), and LEV (300 mg/kg) were injected daily at the same time and seizure stimuli given every alternate day 4-h,1-h, and 30 minutes, respectively, after dosing with above drugs. On day 49, retention trials performed, rats sacrificed, and blood and brains collected for malondialdehyde (MDA) and glutathione (GSH) estimation.

Results: SRT $25 \mathrm{mg} / \mathrm{kg}$ per se showed no protective effect, with SRT and LEV 12.5\% and ESC and SV group none of the rats were kindled. In EPM, kindling increased transfer latency and drug treatments were ineffective. In PA, kindling did not alter transfer latency but SRT alone and SRT + LEV treatment decreased it as compared with baseline $(p<0.001)$. On combining SRT with LEV, opposite effects in MWM and PA were observed. There was a significant decrease in level of MDA in SRT as well as ESC + SV group as compared with normal control on day 49.

Conclusion: Use of antidepressants in PWE has to be done cautiously as their effect on seizure and cognitive impairment may vary.

\section{A0028: "Super-Focal" Cortical Resection in MRI-Negative Epilepsy using Multimodal Imaging-Guided Stereo EEG: A Case Report \\ Moksh Sethi, ${ }^{1,2}$ Patrick Carney, ${ }^{1,1,3}$ Laura K. Zugno, ${ }^{1}$ \\ John Archer, ${ }^{1,2}$ Graeme Jackson ${ }^{1,2}$ \\ ${ }^{1}$ Austin Hospital, Heidelberg, VIC, Australia \\ ${ }^{2}$ The Florey Institute of Neurosciences and Mental Health, \\ Heidelberg, VIC Australia \\ ${ }^{3}$ Eastern Health, Box Hill VIC, Australia}

Objective: To present an epilepsy surgery case report from the Comprehensive Epilepsy Program (CEP) at Austin Health (Heidelberg, Victoria, Australia). In this case, multimodal imaging-guided stereo EEG allowed for a "super-focal" resection adjacent to eloquent cortex with excellent postsurgical outcome.

Methods: Patient records at the Austin Health were reviewed. Clinical details and investigations were summarized. The patient underwent video EEG monitoring, MRI at 3 and 7 Tesla, MEG, PET, SPECT (ictal and interictal), functional-MRI (EEG-fMRI and resting state functional connectivity) prior to proceeding to invasive monitoring using stereo-EEG and electrical stimulation.

Results: All investigations were reviewed at the Comprehensive Epilepsy Program Meeting at Austin health and a plan was formulated for a very limited parietal corticectomy adjacent to eloquent cortex. The patient underwent surgery without suffering a neurological deficit, and had an excellent outcome (Engel class 1B) at 12 months.

Conclusion: In selected cases, excellent outcomes can be achieved using multimodal imaging guided stereotactically 
placed invasive EEG monitoring allowing "super-focal" corticectomy in MRI-negative epilepsy.

A0029: Clinical Profile and Health-Related Quality of Life in Patients with Alcohol-Related Seizures

Muhammad, ${ }^{1}$ Prasanth S.R., ${ }^{1}$ Shaji C.V., ${ }^{1}$ Jithin Raj'

${ }^{1}$ Govt TD Medical College, Alappuzha, Kerala, India

Background: Alcohol-related seizures (ARS) refer to all seizures associated with alcohol use, including alcohol withdrawal seizures (AWS). There is paucity of literature on health-related quality of life (HRQoL) in alcohol-dependent subjects and its relation to clinical pattern of ARS in developing countries.

Objective: To study the clinical profile and HRQoL of patients admitted with alcohol-related seizures in a tertiary care center.

Materials and Methods: In this cross-sectional study, consecutive patients admitted with new onset ARS (from July 2014 to February 2016) were included. Patients with previous diagnosis of epilepsy, with other provoking causes and those not consenting were excluded. Details of drinking pattern were analyzed. Alcohol dependence was identified by Alcohol Use Disorders Identification Test (AUDIT), score more than 8 was taken as positive. HRQOL was assessed by Life Situation Survey (LSS) questionnaire. A video electroencephalography and CT brain (MRI in focal seizures) was done in all patients.

Results: Of the 124 study subjects, all were males. Mean age of the sample was 32.84 years ( \pm 6.37 years). The average daily consumption was $176 \mathrm{~g}( \pm 140 \mathrm{~g})$. Mean duration of alcohol intake was 17.24 years $( \pm 5.24$ years). Nineteen $(15.3 \%)$ patients had a family history of seizure. In focal seizures, MRI revealed symptomatic lesions in three patients. Occurrence of multiple seizures correlated with higher AUDIT scores. Patients with LSS score $<80$ had significantly higher AUDIT score and longer duration of alcohol intake $(p<0.05)$.

Conclusion: A protocol-based evaluation of ARS is necessary, to identify etiologies other than withdrawal. MRI brain is useful in focal onset ARS, to identify underlying symptomatic lesions (33.3\% of partial seizures). Patients with higher AUDIT scores are more likely to have multiple seizures and a poorer HRQOL.

\section{A0030: Awareness and Attitudes of General Physicians' toward Epilepsy Surgery \\ Natesan D., ' Saminathan, ${ }^{1}$ Poovizhi, ${ }^{1}$ Pratheesh Ravindran' 'Department of Neurosurgery, Mahatma Gandhi Medical College and Research Institute, Pondicherry, India}

Objective: Over $30 \%$ of the people with epilepsy $(p \circ W)$ are resistant to medications. There is a significant surgical gap in India as only a minority of candidates for epilepsy surgery is referred for surgical evaluation. We investigated general physicians' views on referral of epilepsy patients for further evaluation and epilepsy surgery.

Materials and Methods: A questionnaire was developed and validated by a pilot study. The electronic questionnaire was sent out to general physicians, practicing in towns of southern India. We collected response from 140 doctors.

Results: Sixty-nine percent of the respondents worked in government hospitals. Eighty-four percent of them felt MRI and EEG were essential for epilepsy evaluation. But 55\% felt CT brain and X-ray were essential for epilepsy evaluation. On commenting about treatment, $85 \%$ felt other modalities in addition to medications were necessary for epilepsy treatment. Psychotherapy was the commonest add-on for antiepileptics. Seventy-six percent felt resective brain surgery was an option for PoW. Only $39 \%$ considered vagal stimulation as a treatment option. Only $24 \%$ of the respondents knew the correct definition of drug-resistant epilepsy. More than two thirds were willing to refer their epilepsy patients to neurologist. Regarding safety of epilepsy surgery safety, $67 \%$ did not have any experience and only $24 \%$ felt it was safe. Only $36 \%$ felt epilepsy surgery had high cost-effectiveness in long-term perspective of eligible patients. Only $11 \%$ felt the epilepsy care in India was adequate and $17 \%$ felt it was grossly inadequate. Forty-seven percent of the physicians felt specialized epilepsy training program to doctors of primary health center and district hospitals would improve epilepsy care in India.

Conclusion: Most of the physicians had good understanding about inadequacies of epilepsy care in India. They felt the need for special training program for improving epilepsy care in India. Uncertainties about benefits of epilepsy surgery among referring general physicians may contribute to the delay in referral to epilepsy surgery.

\section{A0031: Social Cognition Abilities in Patients with Chronic Temporal Lobe Epilepsy \\ Nishanth P.,' Suryaprabha, ${ }^{1}$ Hemalatha, ${ }^{1}$ Rukmini, ${ }^{1}$ Rupam Borgohain ${ }^{1}$ \\ 'Nizam's Institute of Medical Sciences, Hyderabad, Telangana, India}

Background: Social cognition is a high-level cognitive function that includes all the processes that are used to understand and store information about the interactions with other people in a social context. Perception of social signals pertaining to others' mental states is essential to act appropriately in that social context. Low performances in mentalizing abilities on different Theory of Mind (ToM) tasks have been reported in patients with temporal lobe epilepsy (TLE).

Aim: To investigate social cognition abilities in patients with temporal lobe epilepsy.

Materials and Methods:: Thirty-two consecutive patients with TLE ( 19 males; mean age $=27 \pm 11.7$ years) and 30 controls (mean age $=25.24+5.9$ years) are taken into study. Demographic profile, age at onset, ictal semiology, seizure frequency, and response to treatment are studied. All underwent prolonged video EEG monitoring, MRI brain to locate the side of lesion. Affective and cognitive aspects of Faux Pas in the form of 20 stories are assessed in both patients and controls. 